\title{
Estrategias lúdicas para el desarrollo del pensamiento lógico matemático en niños de preparatoria
}

\author{
Cristiana Verónica Gordon Torres ${ }^{1}$ \\ p700232573@ucvvirtual.edu.pe \\ https://orcid.org/0000-0001-5192-2097 \\ Universidad “César Vallejo" Piura - Perú
}

Cesar Balladares Atoche cesarballadares@hotmail.com https://orcid.org/0000-0001-8242-7742 Universidad “César Vallejo” Piura - Perú

Betsy Janeth Bravo Cedeño janethbravo26@yahoo.es https://orcid.org/0000-0002-5875-7968 Universidad “César Vallejo” Piura - Perú

Ludia Marisol Quito Santana p7002325162@ucvvirtual.edu.pe https://orcid.org/0000-0003-4103-6467 Universidad “César Vallejo” Piura - Perú

María Patricia Unuzungo Preciado p7002323567@ucvvirtual.edu.pe https://orcid.org/0000-0001-9372-2712 Universidad “César Vallejo” Piura - Perú

\section{RESUMEN}

El presente estudio se realizó con el objetivo de proponer programa de estrategias lúdicas para el desarrollo del pensamiento lógico matemático en los niños de una institución educativa en Santo Domingo, 2021. El informe metodológicamente de enmarca en el enfoque cuantitativo, de tipo básica, con un diseño no experimental, descriptivopropositivo; la población y muestra estuvo constituida por 80 niños, elegidos de forma no probabilística. Se utizó como técnica de recojo de datos la observación y como instrumento la lista de cotejo, este instrumento paso por los procesos de validez de expertos y confiabilidad con resultados muy favorables. 
De acuerdo a los resultados la variable pensamiento lógico matemático tenemos que el $45 \%$ de los niños se ubican en el nivel medio, además de un $55 \%$ en el nivel alto, estos resultados permiten inferir que en el diagnostico se evidencia que se desarrollan actividades a través de bloque de relaciones y funciones, bloque numérico, bloque geométrico y Bloque de media. Frente a estos resultados se hace necesario la aplicación de la propuesta estrategias lúdicas para el desarrollo del pensamiento lógico matemático en los niños que son parte de la investigación.

Palabras clave: pensamiento lógico; estrategias lúdicas; didáctica; aprendizaje. 


\title{
Playful strategies for the development of mathematical logical thinking in high school children
}

\begin{abstract}
The present study was carried out with the objective of proposing a program of ludic strategies for the development of mathematical logical thinking in children of an educational institution in Santo Domingo, 2021. The methodologically report is framed in the quantitative approach, of a basic type, with a non-experimental, descriptivepurposeful design; the population and sample consisted of 80 children, chosen in a nonprobabilistic way. Observation was used as the data collection technique and the checklist as an instrument, this instrument went through the processes of expert validity and reliability with very favorable results.

According to the results of the variable mathematical logical thinking, we have that $45 \%$ of the children are located in the medium level, in addition to $55 \%$ in the high level, these results allow us to infer that in the diagnosis it is evidenced that activities are developed at through block of relations and functions, number block, geometric block and mean block. Faced with these results, it is necessary to apply the proposed playful strategies for the development of mathematical logical thinking in the children who are part of the research.
\end{abstract}

Keywords: logical thinking; playful strategies; didactics; learning.

Artículo recibido: 30 noviembre. 2021 Aceptado para publicación: 29 diciembre 2021 Correspondencia: fredy.rodríguez@unh.edu.pe Conflictos de Interés: Ninguna que declarar 


\section{INTRODUCCIÓN}

El desarrollo del pensamiento lógico en toda la humanidad, representa una acción importante dentro de su formación integral, pues dentro de las funciones básicas que ejecuta el cerebro, está el saber pensar para resolver los múltiples problemas que a diario sortea el hombre. Unicef, (2019) mediante un informe técnico en los países de américa latina y el caribe han manifestado, existe un $20 \%$ de los estudiantes en edades de 5 a 6 años que encuentran dificultades en la adquisición de competencias relacionadas al pensamiento matemático, asimismo un $30 \%$ de los alumnos de sexto de primaria no logran alcanzar las capacidades, habilidades y el conocimiento mínimo en el área de matemática, al igual que un 53\% secundaria. De acuerdo a estos resultados se puede inferir que mientras se avanza en el nivel de estudios el problema se agudiza de forma más notorio.

Además por lo descrito anteriormente, para el caso de Argentina SITEAL (2020), este organismo después de realizar un diagnóstico para indicar la situación real de los estudiantes en relación al pensamiento lógico en estos tiempos de pandemia considera que un $35 \%$ de la población estudiantil no les dedican tiempo a las matemáticas, esto debido a múltiples factores que van desde el las estrategias de los maestros hasta los niveles de conectividad por el aprendizaje, en ese sentido se plantear alternativas de cambio en la mentalidad de los estudiantes con énfasis en despertar el gusto por la matemática, desarrolla la propuesta "contemos jugando" la misma que pone en práctica el rol de la familia y amistades y el uso de estrategias herramientas lúdicas que orienten el desarrollo de habilidades del pensamiento lógico y la creatividad.

Para el caso de Ecuador, se presentan dificultades grandes y graves de los alumnos ecuatorianos para actuar de forma competente en contextos que movilizan la capacidad de resolución de problemas matemáticos de acuerdo a los resultados de las pruebas PISAD 2018, en las que por primera vez participó Ecuador. El 70,9\% de los alumnos ecuatorianos no alcanzó el nivel 2 en Matemáticas, es decir no logró superar la categoría del nivel de desempeño básico. (Diario el Universo, 2019).

Asimismo, de acuerdo con los resultados de PISA (2020) considera que para los resultados en el contexto ecuatoriano no se puede ignorar, porque las conclusiones son abrumadoras: el $71 \%$ y $51 \%$ de alumnos no supero el nivel básico en el área de matemática. De manera determinante los niños y niñas necesitan aprender buscando 
estrategias idóneas que permitan concordar adecuadamente, el juego, las situaciones lúdicas con la realidad, utilizando materiales concretos, el raciocinio, identificando situaciones de la realidad que ellos viven, equiparando siempre la lógica y la orientación correcta de realizar sus actividades

Asimismo es importante conocer investigaciones en otros contextos que tenga relación con el presente informe, en tal sentido según la búsqueda bibliográfica tenemos antecedentes internacionales, como es el caso de Delgado (2020) investigó sobre “el juego como estrategia para favorecer el concepto de número y la resolución de problemas en un grupo de tercer grado de preescolar". Entre las principales conclusiones se logró que los estudiantes comunicaran los números a través de la palabra oral y escrita, además de manifestar nociones matemáticas como conjugar, comparar y especificar, incluso aplicar la resolución de problemas utilizando el conteo, además que a través de la estrategia del juego, se demostró logros con respecto a la capacidad de resolver problemas numéricos. De la misma manera Poma, Reyes (2019) investigaron sobre "Aplicación de la estrategia de juegos y pensamiento lógico matemático en los estudiantes de 4 años en la I.E. N 004 El mundo de Ana María de Santa Lucía - Uchiza”. De acuerdo al desarrollo de la experiencia se pudo determinar que la aplicación de la estrategia de los juegos mejoro el pensamiento lógico, en este sentido se mejoró el nivel de aprendizaje en donde se pone en evidencia que después de la aplicación del estímulo el grupo experimental en un $24.4 \%$ de los estudiantes se encuentran en un nivel de inicio, asimismo el 48,2\% en logro previsto, inclusive encontramos un 27,4 en logro destacado, teniendo en cuenta estos resultados la aplicación de la estrategia mejoro el pensamiento lógico.

En lo que se refiere al trabajo investigativo, sobre la variable las estrategias lúdicas tenemos el aporte de Díaz y Hernández (2002, p. 234), en el cual indica que "son instrumentos con cuya ayuda se potencian las actividades de aprendizaje y solución de problemas". En este sentido los autores hacen referencia que cuando el docente utiliza este conjunto de estrategias y actividades del juego se pueden lograr resultados muy interesantes en el aprendizaje, en este sentido se modifican la estructura de los contenidos adaptándose a facilitar el aprendizaje, inclusive se concluye que las actividades diseñadas con recursos se planifican en donde se busca una participación actividad por parte del estudiante. 
Por otro lado, tenemos el aporte de García (2004), en donde afirma que las estrategias motivan al estudiante a "exploración y a la investigación en torno a los objetivos, temas y contenidos (p. 80). de igual manera se incorpora recursos lúdicos como imágenes, figuras, sonidos y otros más, que le permiten al estudiante desarrollar un ambiente adecuado y acogedor en donde se pone de manifiesto un estado de confort para generar el interés y sobre todo se sienta motivado por el conocimiento que debe aprender. Para una mejor comprensión de la variable en mención se desintegra en dimensiones entre las cuales de acuerdo a la búsqueda bibliográfica se tiene que en palabras de Calero (2005) se plantean las siguientes: Juegos de discriminación visual, son juegos que permiten al infante lograr la discriminación de las propiedades: color, forma, tamaño de los seres y objetos, además resulta muy importante que el niño realice diversos juegos de clasificación, observación, comparación y correspondencia de las propiedades de los objetos y seres.

Además tenemos la dimensión Juegos de memoria visual, de acuerdo con (Calero, 2005) estos juegos son entretenidos, conformados con diversos colores llamativos, dibujos que expresan diversas imágenes de frutas, animales, juguetes, etc., usando un sistema repetitivo que contribuye al estudio, a la correlación de conceptos y al ejercicio de la memoria; finalmente se considera la dimensión Juegos aritméticos que fomenta en los niños la enseñanza de conocimientos pre matemático utilizando una estrategia lúdica, superando con éxito la asignatura de matemática. Estos juegos son vitales para los niños ya que les permiten descubrir las nociones de magnitudes y sus medidas; también fomenta la habilidad de discriminación entre las diversas figuras geométricas.

En relación a las teorías de la variable estrategias lúdicas se considera el aprendizaje significativo, que de acuerdo a Requena \& Sainz (2009), fundamentan que el aprendizaje significativo se manifiesta en: Desde la perspectiva del niño, existe aprendizaje cuando es capaz de darle sentido y significado; otorgándole significado cuando establece correlación entre sus saberes previos y el aprendizaje nuevo, es decir la relación entre lo que ya conoce y lo que está aprendiendo. Asimismo se considera la teoría del aprendizaje por descubrimiento el mismo que se pone de manifiesto cuando se considera la escuela en el ambiente que propicia el descubrimiento determinando la labor del profesor, el cual presenta situaciones problemáticas para que los estudiantes por descubrimiento obtengan la respuesta, siendo la información esencial: las nociones fundamentales, patrones o 
relaciones de materias. De acuerdo con estas teorías se puede inferir que los niños al jugar exteriorizan sus miedos, angustias, alegrías, intereses, necesidades y la actividad lúdica fomenta el placer, disfrute de resolver problemas de forma significativa, poniendo en práctica diversos procesos mentales y sociales.

En el presente acápite se hace referencia a la variable pensamiento lógico matemático en palabras de Piaget, (1975), es el conocimiento que se construye mediante la solución de problemas; fundamentando que el desarrollo del pensamiento lógico matemático está relacionado con la con la construcción de la noción de número; estableciendo una distinción fundamental entre tres tipos de conocimiento: conocimiento físico, conocimiento lógico matemático y conocimiento social-convencional. En esta perspectiva también se considera el aporte de (Oliveros, 2002, p. 126) en el cual sustenta que el razonamiento lógico es predominantemente deductivo, lo que permite de forma general analizar y encausar situaciones problemáticas que nos presenta la vida en la cotidianeidad.

En relación a las dimensiones del pensamiento lógico se considera lo planteado por Rodríguez (2016) el cual establece cuatro dimensiones para el desarrollo del pensamiento lógico matemático: la dimensión Bloque de relaciones y funciones: este aspecto nace y se desarrolla en los primeros años de su formación escolar con los procesos de descripción, reproducción, construcción de patrones de objetos y figuras, después se empieza a identificar regularidades, reconocer un mismo patrón bajo diferentes procesos y el uso de patrones para predecir valores, cada año se incorpora niveles de complejidad hasta que los estudiantes tengan la capacidad de construir patrones de crecimiento de forma exponencial. (Rodríguez, 2016). La dimensión bloque numérico, implica que se examinan los datos, además de buscar diversas formas de representación (Medina, 2017). Como también se prioriza la relación entre números y la diversidad de sistemas numéricos, ello hace posible la comprensión del significado en las representaciones y además poder identificar la relación entre sí en ese sentido esta dimensión permite desarrollar las actividades con fluidez y sobre todo poder realizar estimaciones de acuerdo a los números (Acosta et al., 2019).

La dimensión bloque geométrico: de acuerdo con Chavarría, et al. (2019) en donde se busca comprender las particularidades y propiedades de las diversas formas y figuras en múltiples dimensiones, además nos permite elaborar problemas matemáticos teniendo en 
cuenta "relaciones geométricas, especificar localizaciones, describir relaciones espaciales, aplicar transformaciones y utilizar simetrías para analizar situaciones matemáticas" (Sezen \& Bülbül, 2018). La dimensión bloque de medida que de acuerdo con Rodríguez (2016) dentro de las exigencias de su cumplimiento se pretende obtener atributos para la medición en los objetos tal es el caso de longitud, peso, capacidad desde las concepciones más básicas, es decir desde la fase inicial pasando por analizar y comprender las unidades de medición y sobre todo poner en práctica para poder ponerlo de manifiesto en su entorno.

Para Piaget (1998) el pensamiento lógico matemático se sustenta en la teoría psicogenética la misma que está estrictamente relacionada al desarrollo cognitivo del niño y que para que se pueda cumplir se deben desarrollar los estadios o subestadios cada uno con sus múltiples manifestaciones, en este contexto de actividades o procedimientos "cada persona de forma independiente se constituyen o preparan entre sí para formar organizaciones de discernimiento" (p. 12).

Por lo antes expuesto se consideró la siguiente interrogante de investigación: ¿Será posible que las estrategias lúdicas desarrollen el pensamiento lógico matemático en los estudiantes de una institución educativa en Santo Domingo, 2021? Además el presente estudio de investigación tuvo como objetivo general: Proponer programa de estrategias lúdicas para el desarrollo del pensamiento lógico matemático en los niños de una institución educativa en Santo Domingo, 2021. Además se plantearon objetivos específicos como son: Diagnosticar el nivel de desarrollo del pensamiento lógico matemático en los niños de una institución educativa en Santo Domingo, 2021; Planificar programa de estrategias lúdicas para el desarrollo del pensamiento lógico matemático en los niños de una institución educativa en Santo Domingo, 2021; Validar programa de estrategias lúdicas para el desarrollo del pensamiento lógico matemático en los niños de una institución educativa en Santo Domingo, 2021.

\section{ESTRATEGIAS METODOLÓGICAS O MATERIALES Y MÉTODOS}

De acuerdo con las características de la investigación, el presente informe es una investigación básica, la cual, de acuerdo con Sánchez, Reyes (1998) se define como una indagación cuyo propósito es obtener una data real con la finalidad de ampliar los conocimientos científicos, descubriendo nuevas teorías. Según Caballero (2014) las investigaciones descriptivas tienen como condiciones conseguir el eje dominante 
cuantitativo, el cual emplea un método detallado y narrativo logrando definir las características del fenómeno observado (p.100). También se centra en el tipo propositivo, es por ello que según por Hurtado de Barrera (2008) indica que la indagación propositiva o prospectiva se orienta a formular propuestas resolutivas frente a determinados contextos, para ello es indispensable partir de la exploración. Se consideró el diseño no experimental, descriptivo-propositivo, previo es indispensable señalar que no existió manipulación de la variable

Para el recojo de los datos se utilizó la técnica de la observación. Según Valderrama, León (2009) la define como un método clásico que permite la obtención de data en la cual se visualiza la cualidad externa e interna de un elemento o nivel de conducta y procedimiento de un individuo o individuos. Con respecto al instrumento utilizado para recolectar la data de la variable pensamiento lógico matemático la lista de cotejo que de acuerdo con Valderrama (2013) consiste en un cuadro de doble entrada en donde en la parte horizontal se consigna el nombre de las acciones a observar, es decir todo aquello que se necesita recoger como datos. La misma que fue construida para medir el nivel de pensamiento lógico matemático con sus dimensiones: Bloque de relaciones y funciones, bloque numérico, bloque geométrico, bloque de media. Estos instrumentos fueron validados por juicios de expertos, los mismos que son profesionales probos en la materia de investigación, concluyendo validez de contenido aceptable; además se utilizó el coeficiente Alfa de Cronbach, para determinar la fiabilidad del instrumento el mismo que fue de 0,90 .

Por otro lado, tenemos una muestra que estuvo constituida por 80 niños que pertenecen a la institución educativa objeto de investigación; esta muestra de estudio fue elegido de manera no probabilística a criterio del investigador. (Ary et al., 1989). Por otro lado, en relación al procesamiento de los datos se utilizó la estadística descriptiva, la primera para promediar los valores de la variable y las dimensiones.

\section{RESULTADOS Y DISCUSIÓN}


Los resultados fueron recogidos a través de lista de cotejo, lógicamente las preguntas están orientadas a la consecución del objetivo general, en donde se obtuvieron los siguientes datos:

\section{Tabla 1}

Nivel pensamiento lógico matemático

\begin{tabular}{ccc}
\hline Nivel & Frecuencia & Porcentaje \\
\hline Medio & 36 & 45,0 \\
Alto & 44 & 55,0 \\
\hline Total & $\mathbf{8 0}$ & $\mathbf{1 0 0 , 0}$
\end{tabular}

Nota. En la Tabla 1 se presentan los datos recogidos a través de la lista de cotejo a los niños de Santo Domingo - 2021

Según los datos que se muestran en la Tabla 1 tenemos que el $45 \%$ de los niños observados en relación a la variable pensamiento lógico matemático se ubican en el nivel medio, asimismo se evidencia un $55 \%$ en el nivel alto. Estos resultados se muestran toda vez que los niños desarrollan actividades que comprenden clasificación - seriación, ecuaciones, resolver problemas, sistemas de medición, etc.

\section{Tabla 2}

Nivel bloque de relaciones y funciones

\begin{tabular}{ccc}
\hline Nivel & Frecuencia & Porcentaje \\
\hline Bajo & 13 & 16,3 \\
Medio & 60 & 75,0 \\
Alto & 7 & 8,8 \\
\hline Total & $\mathbf{8 0}$ & $\mathbf{1 0 0 , 0}$ \\
\hline
\end{tabular}

Nota. En la Tabla 2 se presentan los datos recogidos a través de la lista de cotejo a los niños de Santo Domingo - 2021

De acuerdo a la Tabla 2 tenemos que el $16.3 \%$ de los niños observados en relación a la dimensión bloque de relaciones y funciones se ubican en el nivel bajo, asimismo se evidencia un $75.0 \%$ en el nivel medio, además de un $8.8 \%$ está ubicado en el nivel alto. Estos datos son producto de las actividades que realizan los niños como son comparar y explicar situaciones de la vida diaria.

\section{Tabla 3}


Nivel bloque numérico

\begin{tabular}{ccc}
\hline Nivel & Frecuencia & Porcentaje \\
\hline Bajo & 17 & 21,3 \\
Medio & 60 & 75,0 \\
Alto & 3 & 3,8 \\
\hline Total & $\mathbf{8 0}$ & $\mathbf{1 0 0 , 0}$
\end{tabular}

Nota. En la Tabla 3 se presentan los datos recogidos a través de la lista de cotejo a los niños de Santo Domingo - 2021

Según los datos que se registran en la Tabla 3 tenemos que el 21,3\% de los niños observados en relación a la dimensión bloque numérico se ubican en el nivel bajo, asimismo se evidencia un $75.0 \%$ en el nivel medio, además de un 3.8\% está ubicado en el nivel alto. Estos resultados se muestran toda vez que los niños se les solicita expresa oralmente los números ordinales, explica con su propio lenguaje sobre desplazamientos o recorridos a partir de una experiencia vivencial o lúdica, etc.

\section{Tabla 4}

Nivel bloque geométrico

\begin{tabular}{ccc}
\hline Nivel & Frecuencia & Porcentaje \\
\hline Bajo & 15 & 18,8 \\
Medio & 54 & 67,5 \\
Alto & 11 & 13,8 \\
\hline Total & $\mathbf{8 0}$ & $\mathbf{1 0 0 , 0}$
\end{tabular}

Nota. En la Tabla 4 se presentan los datos recogidos a través de la lista de cotejo a los niños de Santo Domingo - 2021

Según los datos que se muestran en la Tabla 4 tenemos que el 18,8\% de los niños observados en relación a la dimensión bloque geométrico se ubican en el nivel bajo, asimismo se evidencia un $65.5 \%$ en el nivel medio, además de un $13.8 \%$ está ubicado en el nivel alto. Estos resultados demuestran que los niños esporádicamente se les solicita identifiquen características perceptuales de los objetos de su entorno, además de explicar su ubicación, asimismo encontramos desarrollado de actividades como identificar al lado de, cerca de, lejos de, esto le permite tener una idea razonable sobre el conocimiento geométrico.

\section{Tabla 5}


Nivel bloque de medida

\begin{tabular}{ccc}
\hline Nivel & Frecuencia & Porcentaje \\
\hline Medio & 31 & 38,8 \\
Alto & 49 & 61,3 \\
\hline Total & $\mathbf{8 0}$ & $\mathbf{1 0 0 , 0}$
\end{tabular}

Nota. En la Tabla 5 se presentan los datos recogidos a través de la lista de cotejo a los niños de Santo Domingo - 2021

Según los datos que se muestran en la Tabla 5 tenemos que el 38,8\% de los niños observados en relación a la dimensión bloque de medida se ubican en el nivel medio, asimismo se evidencia un $61.3 \%$ en el nivel alto. Estos resultados se muestran toda vez que los niños desarrollan la capacidad de comparar dos objetos y expresa la longitud utilizando su propio lenguaje.

Frente a estos resultados tenemos el aporte teórico de Piaget (1998) en donde considera que el desarrollo cognitivo del niño se deben desarrollar los estadios o subestadios cada uno con sus múltiples manifestaciones, en este contexto de acción o conceptuales de "cada persona se organizan o combinan entre sí hasta formar estructuras de conocimiento". Con estos argumentos tenemos lo planteado en la Tabla 1 en donde indica que los niños en un $45 \%$ responden a un nivel medio, asimismo se evidencia un $55 \%$ en el nivel alto.

Estos resultados se complementan con lo planteado por Paltan, Quilli (2016) en el cual indica que los estudiantes desarrollaron la habilidad de analizar y argumentar los pasos, fases y procedimientos para la resolución de problemas, esto se manifiesta en que un $67 \%$ mejoro dichas habilidades, por esta razón desarrollan estrategias para favorecer el aprendizaje de los niños cual le permite mejorar su forma de entender los problemas matemáticos y sobre todo poner de manifiesto estas habilidades para entender $\mathrm{y}$ comprender situaciones que le tocan experimentar en su vida cotidiana.

\section{CONCLUSIÓN O CONSIDERACIONES FINALES}

La variable pensamiento lógico matemático tenemos que el $45 \%$ de los niños se ubican en el nivel medio, además de un 55\% en el nivel alto, estos resultados permiten inferir que en el diagnostico se evidencia que se desarrollan actividades a través de bloque de relaciones y funciones, bloque numérico, bloque geométrico y Bloque de media.

La dimensión bloque de relaciones y funciones, el mismo que implica manejo de nociones básicas de clasificación, seriación y ecuaciones sucesivas manifiestan que el 16.3\% de los 
niños se ubican en el nivel bajo, es decir no cumplen con estas destrezas de realizar sucesiones, ordenar, comparar cantidades etc., un $75.0 \%$ se considera en el nivel medio debido a que se realizan estas actividades, pero no lo hacen de forma en que todos participen.

Para la dimensión bloque numérico tenemos que el 21,3\% de los niños se ubican en el nivel bajo, además tenemos que un $75.0 \%$ en el nivel medio, estos datos nos permiteN inferir que los estudiantes realizan procedimientos para poder desarrollar conteo en material concreto, y resolución de problemas, pero también se evidencia la falta de conocimiento matemático y la habilidad de resolver problemas.

La dimensión bloque geométrico tenemos que el 18,8\% de los niños se ubican en el nivel bajo, un $65.5 \%$ en el nivel medio, esto nos permite inferir que los niños presentan dificultades para relacionar formas y figuras, además de no establecer argumentos matemáticos, presenta las dificultades de ubicación del razonamiento espacial; se destaca un $13.8 \%$ está ubicado en el nivel alto es decir cumple con las actividades planteadas.

La dimensión bloque de medida tenemos que el 38,7\% de los niños se ubican en el nivel medio es decir presentan dificultades para comparar datos de longitud, capacidad y peso, sistemas de medición y Resolución de problemas, asimismo tenemos un $61.3 \%$ en el nivel alto, es decir cumple dichas actividades

\section{LISTA DE REFERENCIAS}

Acosta, G.; Rivera, L.; Acosta, M. (2019). Desarrollo del Pensamiento Lógico Matemático. https://www.sammateo.edu.co/documentos/publicaciondesarrollo-pensamiento-logico.pdf

Albújar, S. (2020). Habilidades de precálculo en estudiantes de cinco años en una institución educativa inicial pública de mi Perú. http://repositorio.usil.edu.pe/bitstream/USIL/10431/1/2020_Alb\%C3\%BAjar\%2 0N\%C3\%BA\%C3\%B1ez.pdf

Alsina, A. (2015). Como desarrollar el pensamiento matemático de 0 a 6 años. Barcelona: OCTAEDRO.

Batanero, C. (2016). La comprensión de la probabilidad en los niños: ¿qué podemos aprender de la investigación? https://www.researchgate.net/publication/273456653_LA_COMPRENSION\%2 0N\%C3\%BA\%C3\%B1ez.pdf_DE_LA_PROBABILIDAD_EN_LOS_NINOS_ 
QUE_PODEMOS_APRENDER_DE_LA_INVESTIGACION_PDFA.pdf?sequ ence $=5 \&$ isAllowed $=\mathrm{y}$

Benítez, P. (2019). Influencia de los juegos matemáticos con el uso de las TICs en el desarrollo del pensamiento lógico matemático en párvulos. http://www.redisd.org/index.php/es/resumen-recibidos-mt2/932-influencia-delos-juegos-matematicos-con-el-uso-de-las-tics-en-el-desarrollo-del-pensamientologico-matematico-en-parvulos-2

Bohumil, F. (2015). An Introduction to Fictional Worlds Theory. https://www.peterlang.com/document/1050008

Calero, M. (2005). Educar jugando. Lima, Perú: Alfaomega.

Camana, R. (2021, marzo 10). Aprender matemáticas con el 'Super Héroe Doctor. https://www.educaciontrespuntocero.com/experiencias/aprender-matematicasheroes-marvel/

Carrasco, S. (2009). Metodología de la investigación científica. Lima, Perú: San Marcos.

Chavarría, S., Novoa, P., Sánchez, F., Uribe, Y. \& Ramírez, Y. (2019). Funciones ejecutivas y nociones matemáticas en prescolares de cinco años. Scientific Journal of Education - $\quad$ EDUSER, 6(3). http://revistas.ucv.edu.pe/index.php/EDUSER/article/view/2416

Cortes, A; Saldías, M. (2004). Estudio del concepto de sublimación en la obra de Sigmund Freud. https://repositorio.uchile.cl/bitstream/handle/2250/106410/cortes_a.pdf?sequence $=3 \&$ is Allowed $=\mathrm{y}$

Delgado, C. (2020). El juego como estrategia para favorecer el concepto de número y la resolución de problemas en un grupo de tercer grado de preescolar. https://repositorio.tec.mx/bitstream/handle/11285/636366/DelgadoVazquez

Diario el Universo (2019, febrero 26). Ecuador reprobó en Matemáticas en evaluación internacional. https://www.eluniverso.com/guayaquil/2019/02/26/nota/7207946/matematic asno-se-paso-prueba/

Díaz, F. y Hernández, G. (2002). Estrategias docentes para un aprendizaje significativo: una interpretación constructivista. Mc Graw Hill. México. 
Ferreira, H. \& Pedrazzi, G. (2007). Teorías y enfoques psicoeducativos de aprendizaje. Argentina: Noveduc.

Ferreiro, R. (2009). Estrategias didácticas del aprendizaje cooperativo. México. Editorial Trillas.

Fulleda, P. (2003). Lúdica por el desarrollo humano: Programa general de acciones recreativas para adolescentes, jóvenes y adultos. http://www.redcreacion.org/documentos/simposio3vg/PFulleda.html

Gamarra, G., Berrospi, J., Pujay, O., Cuevas, R. (2008). Estadística e investigación. Lima. Perú: Editorial San Marcos.

García, J. (2004). Ambientes con recursos tecnológicos. Costa Rica. Editorial EUNED.

Godoy, C., Abad, M., Torres, F. (2020). Gamification in the development of mathematical logical thinking in university students. https://orcid.org/00000001$5505-7715$

Godoy, E. (2020). Gamificación en el desarrollo del pensamiento lógico matemático en universitarios. 3C TIC. Cuadernos de desarrollo aplicados a las TIC, 9(3), 107145. https://doi.org/10.17993/3ctic.2020.93.107-145

Guzmán, P. (2019). El razonamiento lógico matemático y su influencia en el rendimiento académico en Matemática I de los estudiantes del primer ciclo de una Universidad Privada, 2018. (Tesis doctoral, Universidad Educación Enrique Guzmán y Valle). https://repositorio.une.edu.pe/bitstream/handle/UNE/3044/TM\%20CE-

Hernández, R. Zapata, N., Mendoza, C. (2013). Metodología de la investigación para bachillerato. Enfoque por competencias. México: McGraw-Hill/Interamericana Editores.

Hernández, R., Mendoza, C. (2018). Metodología de la investigación. Las rutas cuantitativa, cualitativa y mixta. México: McGraw-Hill/Interamericana Editores.

Hidalgo, D. (2021, abril 14). Docentes de la SEG implementan actividades para evaluar el pensamiento matemático en alumnos. https://boletines.guanajuato.gob.mx/2021/04/27/docentes-de-lasegimplementan-actividades-para-evaluar-el-pensamiento-matematicoenalumnos/ 
Hughes, P. Fergus, G. (2006), El juego. Su importancia en el desarrollo psicobiológico del niño y el adolescente. México, Trillas.

Hurtado de Barrera, J. (2008). Metodología de la investigación, una comprensión holística. Investigación proyectiva. http://investigacionholistica.blogspot.com/2008/02/lainvestigacinproyectiva.html

Jaramillo, L.; Puga, L. (2016). Logical - abstract thought as support to boost cognitive processes in education. DOI: 10.17163/soph.n21.2016.01

Jiménez, M. (2004). Jugar: la forma más divertida de educar. España. Ediciones

Latorre, A.; Delio del Rincón, I.; Arnal, J. (1996). Bases metodológicas de la investigación educativa. Barcelona: GR92.

Markovits, H.; Luc de Chantal, P.; Brisson, J.; Dubé, E.; Thompson, V.; Newman, I. (2020). Reasoning strategies predict use of very fast logical reasoning. DOI: 10.3758 / s13421-020-01108-3

Medina, M. (2017). Estrategias metodológicas para el desarrollo del pensamiento lógico matemático. Didáctica y Educación, IX(1), 125-132. https://dialnet.unirioja.es/servlet/articulo?codigo $=6595073$

Ministerio de educación (2017). Rutas de aprendizaje. ¿Qué y cómo aprenden nuestros niños y niñas? Desarrollo del Pensamiento Matemático 3, 4 y 5 años de Educación Inicial. https://www.minam.gob.pe/proyecolegios/Curso/cursovirtual/Modulos/modulo2 /web-cambiemoslaeducacion/docs2inicial/Fasciculo-

InicialMatematica.pdf?f=/repositorio/descargas/rutas-2013/Fasciculo-InicialMatematica.pdf

Montoya, M. (2020). Didactic strategies for the development of logicalmathematical thinking in early education children from 4 to 6 years. https://www.grupocieg.org/archivos_revista/Ed.48(115-124)-

Montoya\%20Daniela_articulo_id750.pdf

Oliveros, E. (2002). Metodología de la enseñanza de Matemática. Ed. Santillana.

Oyanguren, J. (2018). El maltrato Infantil y su relación con el pensamiento lógico matemático y habilidades sociales de los niños del tercer ciclo de primaria en la Institución Educativa Robert Letourneau, Los Olivos -2018. (Tesis doctoral, 
Universidad

César

Vallejo).

https://repositorio.ucv.edu.pe/bitstream/handle/20.500.12692/22482/Oyang

uren_AJR.pdf?sequence $=1 \&$ isAllowed=y Palabra. S.A

Paltan, G.; Quilli, K. (2016). Estrategias metodológicas para desarrollar el razonamiento lógico - matemático en los niños y niñas del cuarto año de educación básica de la escuela "Martín Welte" del Cantón Cuenca. (Tesis doctoral, Universidad de Cuenca). Ecuador.

Parra, S. (2020). Resultados Educativos en el Ecuador: Examen Crítico a la Luz de los Exámenes Internacionales

PISA. https://informativo.usfq.com/images/files/Koyuntura\%20Feb\%202020.pdf

Piaget, J. (1975). El mecanismo del desarrollo mental. Madrid, España: Editora Nacional.

Piaget, J. (1979). La abstracción de las relaciones lógico-matemáticas. Buenos Aires: Huemul.

Piaget, J. (1980). Psicología y pedagogía. Barcelona: Ariel.

Pisa. (2020). Resultados Educativos en el Ecuador: Examen Crítico a la Luz de los Exámenes Internacionales PISA.

Poma, I., Reyes, M. (2019). Aplicación de la estrategia de juegos y pensamiento lógico matemático en los estudiantes de 4 años, II nivel de Inicial de las secciones $\begin{array}{lllllll}\text { creativos } y & \text { líderes } & \text { de } & \text { la } & \text { I.E. }\end{array}$ https://repositorio.ucv.edu.pe/handle/20.500.12692/35336

Ramos, C. (2015). Los paradigmas de la investigación científica: DOI: https://doi.org/10.33539/avpsicol.2015.v23n1.167

Requena, M., \& Sainz, P. (2009). Didáctica de la educación infantil. España: Editex.

Reyes, T. (2015). Aplicación de las estrategias lúdicas en el aprendizaje de la lectura en los niños de Educación Primaria. (Tesis doctoral, Universidad Nacional Abierta). Universidad de Córdoba, Isla de Margarita, Venezuela. https://helvia.uco.es/xmlui/bitstream/handle/10396/13789/2016000001489.p d f?sequence $=1$

Rivero, Y. (2012). La planificación de estrategias didácticas y el desarrollo del pensamiento lógico-matemático en educación primaria. División de estudios para graduados. Universidad del Zulia (Venezuela). https://docplayer.es/93750173-La- 
planificacion-de-estrategias-didacticas-y-el-desarrollo-del-pensamiento-logicomatematico-en-educacion-primaria-autor-lcda.html

Rodríguez, M. (2016). El juego en la etapa de educación infantil (3 - 6 años): El juego social. Universidad de Valladolid.

Salvador, A. (2020). El juego como recurso didáctico en el aula de matemáticas. (http://www2.caminos.upm.es/Departamentos/matematicas/grupomaic/conferen cias/12.Juego.pdf

Sánchez, B., Chamoso, J., Rodríguez, M. (2014). El dominó para aprender matemáticas. https://www.researchgate.net/publication/280565204_El_domino_para_aprende r_matematicas

Sánchez, H., Reyes, C. (1998). Metodología y diseño en la investigación científica. Primera reimpresión .Lima -Perú. Editorial Mantaro.

Sezen, N.; Bülbül, A. (2018). A scale on logical thinking abilities https://doi.org/10.1016/j.sbspro.2011.04.131

SITEAL (2020). Programa nacional de matemáticas.

https://siteal.iiep.unesco.org/bdnp/3418/programa-nacional-matematica

Sophian, C. (2016). El conocimiento de las matemáticas en la primera infancia. www.enciclopedia-infantes.com/matematicas/segun-los-expertos/elconocimiento-de-las-matematicas-en-la-primera-infancia.

UNESCO (2021). Las Matemáticas, enseñanza e investigación para enfrentar los desafios de estos tiempos. https://es.unesco.org/news/matematicasensenanza-einvestigacion-enfrentar-desafios-estos-tiempos

Valderrama, S. (2013). Pasos para elaborar proyectos de investigación científica. Cuantitativa, cualitativa y mixta. Segunda edición. Lima, Perú: Editorial San Marcos.

Valderrama, S., León, L. (2009). Técnicas e instrumentos para la obtención de datos en la investigación científica. Lima: Editorial San Marcos.

Vygotsky. L. (1999). Imaginación y creación en la edad infantil. La Habana: Pueblo y Educación.

Yancce, A. (2019). Estrategias lúdicas para la producción de textos narrativos en estudiantes del quinto ciclo de educación primaria, Ayacucho 2019. 
https://repositorio.ucv.edu.pe/bitstream/handle/20.500.12692/44128/Yancce_M A-SD.pdf?sequence $=1 \&$ is Allowed $=\mathrm{y}$

Zulay, N. (2021). Estrategias lúdicas dirigidas a la enseñanza de la matemática a nivel de Educación Primaria. (Tesis de maestría, Universidad de Carabobo, Venezuela). https://doi.org/10.33996/merito.v2i6.261

Zunshine, L. (2016). Why We Read Fiction: Theory of Mind and the Novel. https://www.goodreads.com/book/show/854255.Why_We_Read_Fiction 